\title{
Table of Contents, Volume 8, 2003
}

\section{Original Articles and Reviews}

1 Editorial: Critical Descriptions of Doctoral Studies in Psychology: Preface to Eight Reports

Floyd W. Rudmin

3 Doctoral Training of Psychology Students in France Christine Roland-Lévy

9 Doctoral Training in the French- Speaking Countries of Europe: Objectives and Suggestions for Improvement

Markus Brauer, Jean-Claude Abric, Ewa Drozda-

Senkowska, Patrick Lemaire, Fabio Lorenzi-Cioldi,

Paula Niedenthal, Rasyid Bo Sanitioso, Georges

Schadron, Dirk Steiner, and Vincent Yzerbyt

18 The Training of Doctoral Students of Psychology in Germany

Gerhard Strube and Hans Spada

23 Doctoral Training in Psychology in Italy

Guido Cimino and Antonio M. Ferreri

28 Doctoral Studies in Psychology in Spain Yolanda García Rodríguez

34 The Education of Research Psychologists in Sweden Knut Larsson and Josef Frischer

39 A UK PhD in Psychology: The British Psychological Society Guidelines Jonathan K. Foster

48 The Training of Doctoral Students of Psychology in the United States

David H. Silvera, Bruno Laeng, and Tove I. Dahl

65 Editorial

Kurt Pawlik

66 Psychologists Can Do Much to Support Sustainable Development

Peter Schmuck and Charles Vlek

77 Anomalies in Cognition: Olfactory Memory

Gesualdo M. Zucco
87 Geropsychology and Psychology in the Last Quarter of the 20th Century: Bibliometrical Results for the German-Speaking Versus Anglo-American Research Community

Günter Krampen and Hans-Werner Wahl

92 Searching for Information About Financial Decisions in Euro Versus DM

Eva Jonas and Dieter Frey

97 What Does Human Development Index Rating Mean in Terms of Individualism/Collectivism? Maria José Sotelo and Luis Gimeno

101 Adults' Knowledge of General Psychology Adrian Furnham, Inés Callahan, and Richard Rawles

221 Editorial Kurt Pawlik

223 Childhood Socio-Emotional Characteristics as Antecedents of Marital Stability and Quality Ulla Kinnunen and Lea Pulkkinen

238 Russian Citizens' Representations of the Country's Position in the Geopolitical Space of the Commonwealth of Independent States, Europe, and the World Victor F. Petrenko, Olga V. Mitina, and Kirill A. Bertnikov

252 Multidimensional Anxiety and Content-specificity Effects in Preferential Processing of Threat Manuel G. Calvo, P. Avero, M. Dolores Castillo, and Juan J. Miguel-Tobal

266 Neglecting the Risks: The Irrationality of Health Behavior and the Quest for La Dolce Vita Lennart Sjöberg

279 Extraversion and Neuroticism: The Typical Profiles of Self-Regulation Varvara I. Morossanova 


\section{Special Issue: \\ Psychology of Aging in Europe \\ Rocío Fernández-Ballesteros and Mariagiovanna Caprara, Guest Editors}

129 Editorial: Psychology of Aging in Europe: Rocío Fernández-Ballesteros and Mariagiovanna Caprara

131 Personality's Correlates of Adult Development and Aging Gian Vittorio Caprara, Mariagiovanna Caprara, and Patrizia Steca

148 Cognitive Plasticity in Healthy, Mild Cognitive Impairment (MCI) Subjects and Alzheimer's Disease Patients: A Research Project in Spain Rocío Fernández-Ballesteros, María Dolores Zamarrón, Lluís Tárraga, Rosa Moya, and Julio Iñiguez

160 Psychosocial Profile of Rural and Urban Elders in Portugal

Constança Paúl, António M. Fonseca, Ignácio Martín, and João Amado

168 A German Research Program on the Psychosocial Adaptation to Age-Related Vision Impairment: Recent Findings Based on a Control Theory Approach Hans-Werner Wahl, Oliver Schilling, Stefanie Becker, and David Burmedi

178 Subjective Quality of Life in Old Age in Greece: The Effect of Demographic Factors, Emotional State, and Adaptation to Aging

Anastasia Efklides, Maria Kalaitzidou, and Grace Chankin

192 Life-course Dynamics: A Research Program in Progress from The Netherlands Johannes J.F. Schroots

200 From Variables to Lives: Inputs to a Fresh Agenda for Psychological Aging Research in Norway Svein Olav Daatland

\section{Reports}

54 Clinical Psychology in Spain as Sound as a Bell José M. Prieto and Manuel Berdullas

117 Eurocentric Bias in Women's Psychology Journals: Resistance to Issues Significant to People of Color Ronald E. Hall

\section{News and Announcements}

58, 123, 208, 289 EFPA News

60, 126, 218, 292 Calendar

63 Change of Editor-in-Chief / Call for Nominations for Editor-in-Chief and Associate Editors

294 Reviewers 2002/2003

295 Table of Contents / Keywords / Author Index, Volume 8, 2003

296 Erratum

\section{Erratum}

Caprara, G.V., Caprara, M., \& Steca, P. (2003). Personality's Correlates of Adult Development and Aging. European Psychologist, 8, 131-147.

Tables 1 (p. 135) and 2 (p. 137) in the above-mentioned paper contain columns labeled "Consciousness." They should rather read "Conscientiousness." The authors apologize for any inconvenience caused. 


\section{Keywords, Volume 8, 2003}

$\begin{array}{lrl}\text { Addiction } & 266 & \text { Financial decisions } \\ \text { Admission 131,160, 178, 192, } 200 & 48 & \text { France } \\ \text { Age-related visual impairment } & 168 & \text { Geopolitics } \\ \text { Aging Ang -Anglo-American } \\ \text { Alzheimer's disease } & 148 & \text { comparison } \\ \text { Anxiety } & 252 & \text { Germany } \\ \text { Apprenticeship } & 34 & \text { Gerontology } \\ \text { Attentional bias } & 252 & \text { Gerontopsychology } \\ \text { Attitudes toward currencies } & 82 & \text { Graduate school } \\ \text { Autobiographical memory } & 192 & \text { Graduate student } \\ \text { Behavior change strategies } & 66 & \text { Graduate training } \\ \text { Bibliometrical analysis } & 87 & \text { Happiness } \\ \text { Big Five } & 131 & \text { Higher education } \\ \text { Brain plasticity } & 148 & \text { History of psychology } \\ \text { Cognitive plasticity } & 148 & \text { Human development } \\ \text { Consciousness } & 77 & \text { Human values } \\ \text { Denial } & 266 & \text { Individual differences } \\ \text { Developmental psychology } & 87 & \text { Individualism } \\ \text { Dissertation } & 9 & \text { Information search } \\ \text { Dissonance theory } & 82 & \text { Intelligence } \\ \text { Diversity } & 200 & \text { Interpretative bias } \\ \text { Doctoral degree } & 23,48 & \text { Italy } \\ \text { Doctoral education } & 34 & \text { Learning potential } \\ \text { Doctoral programs } & 18 & \text { Legislation } \\ \text { Doctoral studies } & 28 & \text { Life events } \\ \text { Doctoral training } & 3,9 & \text { Life satisfaction } \\ \text { Dynamic assessment } & 148 & \text { Lifespan psychology } \\ \text { Dynamics } & 192 & \text { Marital instability } \\ \text { Environmental psychology } & 66 & \text { Marital quality } \\ \text { Euro } & 82 & \text { Memory bias } \\ \text { Extraversion } & 279 & \text { Mild cognitive impairment } \\ & & \end{array}$

$\begin{array}{rlr}82 & \text { Morale } & 178 \\ 3 & \text { Multidimensional } & 252 \\ 238 & \text { Neuroticism } & 279 \\ & \text { Odor memory } & 77 \\ 87 & \text { Personality } & 223 \\ 18 & \text { PhD } & 9 \\ 200 & \text { Political map } & 238 \\ 87 & \text { Psychological control } & 168 \\ 9 & \text { Psychology } & 3 \\ 48 & \text { Psychosemantics } & 238 \\ 18 & \text { Psychosocial adaptation } & 168 \\ 178 & \text { Risk perception } & 266 \\ 23,48 & \text { Self-efficacy } & 131 \\ 87 & \text { Self-mechanisms } & 200 \\ 101 & \text { Self-regulation } & 279 \\ 101 & \text { Semantic space } & 238 \\ 279 & \text { Social network } & 160 \\ 101 & \text { Social-emotional characteristics } & 223 \\ 82 & \text { Standardization “learning-alliance “ } & 34 \\ 200 & \text { Stereotypes } & 238 \\ 252 & \text { Strategies and interferences } & 77 \\ 23 & \text { Student supervision } & 28,48 \\ 148 & \text { Subjective well-being } & 178 \\ 28 & \text { Supervisor } & 9 \\ 192 & \text { Sustainable development } & 66 \\ 160,178 & \text { Testing-the-limits } & 148 \\ 192 & \text { Urban/rural } & 160 \\ 223 & \text { Values } & 131 \\ 223 & \text { Well-being } & 131 \\ 252 & & \\ 148 & \text { lal } & \end{array}$




\section{Author Index, Volume 8, 2003}

\author{
Amado, João \\ Avero, P. \\ Becker, Stefanie \\ Berdullas, Manuel \\ Bertnikov, Kirill A. \\ Burmedi, David \\ Caprara, Gian Vittorio \\ Caprara, Mariagiovanna \\ Callahan, Inés \\ Calvo, Manuel G. \\ Castillo, M. Dolores \\ Chankin, Grace \\ Cimino, Guido \\ Daatland, Svein Olav \\ Dahl, Tove I. \\ Efklides, Anastasia \\ Fernández-Ballesteros, Rocío \\ Ferreri, Antonio \\ Fonseca, António M. \\ Foster, Jonathan K. \\ Frey, Dieter
}

$\begin{aligned} 160 & \text { Frischer, Josef } \\ 252 & \text { Furnham, Adrian } \\ 168 & \text { Gimeno, Luis } \\ 54 & \text { Hall, Ronald E. } \\ 238 & \text { Iñiguez, Julio } \\ 168 & \text { Jonas, Eva } \\ 131 & \text { Kalaitzidou, Maria } \\ 129,131 & \text { Kinnunen, Ulla } \\ 101 & \text { Krampen, Günter } \\ 252 & \text { Laeng, Bruno } \\ 252 & \text { Larsson, Knut } \\ 178 & \text { Martín, Ignácio } \\ 23 & \text { Miguel-Tobal, Juan J. } \\ 200 & \text { Mitina, Olga V. } \\ 48 & \text { Morossanova, Varvara I. } \\ 178 & \text { Paúl, Constança } \\ 148 & \text { Pawlik, Kurt } \\ 23 & \text { Petrenko, Victor F. } \\ 160 & \text { Prieto, José M. } \\ 39 & \text { Pulkkinen, Leo } \\ 92 & \text { Rawles, Richard }\end{aligned}$

$\begin{aligned} 34 & \text { Rodríguez, Yolanda García } \\ 101 & \text { Roland-Lévy, Christine } \\ 97 & \text { Rudmin, Floyd W. } \\ 117 & \text { Schadron, Georges } \\ 148 & \text { Schilling, Oliver } \\ 92 & \text { Schmuck, Peter } \\ 178 & \text { Schroots, Johannes J.F. } \\ 223 & \text { Silvera, David H. } \\ 87 & \text { Sjöberg, Leonart } \\ 48 & \text { Sotelo, Maria José } \\ 34 & \text { Spada, Hans } \\ 160 & \text { Steca, Patrizia } \\ 252 & \text { Steiner, Dirk } \\ 238 & \text { Strube, Gerhard } \\ 279 & \text { Tárraga, Lluís } \\ 160 & \text { Vlek, Charles } \\ 221 & \text { Wahl, Hans-Werner } \\ 238 & \text { Yzerbyt, Vincent } \\ 54 & \text { Zamarrón, María Dolores } \\ 223 & \text { Zucco, Gesualdo M. }\end{aligned}$

28

3

1 